\section{Ausgezeichnet: Beste Kasuistik 2017}

Der diesjährige Kasuistikpreis der Zeitschrift Pneumologie geht an Dr. Stefanie Keymel vom Universitätsklinikum Düsseldorf für den Fallbericht „Bronchitis plastica - Akute Luftnot und bronchiale Ausgusspräparate“. Der mit $\mathbf{5 0 0}$ Euro dotierte Preis wurde auf dem DGP-Kongress in Dresden verliehen.

Bei der Wahl der ausgezeichneten Arbeit war sich die Jury, bestehend aus den Herausgebern der Zeitschrift Prof. Tom Schaberg und Prof. Santiago Ewig, schnell einig. In seiner Laudatio verwies Prof. Ewig auf die Bedeutsamkeit der Publikation: „Die Kasuistik zeichnet sich dadurch aus, dass durch ein einziges markantes Bild eine Krankheit in einer Weise charakterisiert wird, die man nicht wieder vergisst. Der Text ist zugespitzt auf die wichtigsten klinischen Daten. Man ist geneigt, den Fallbericht zweimal hintereinander zu lesen. So prägen sich auch seltene Erkrankungen gut ein. Man wird sie erkennen, auch wenn man sie selbst noch nie vorher gesehen hat.“
„Der kniffelige Fall mit den Leitsymptomen Husten und Luftnot bei einer komplex erkrankten Patientin erforderte Teamarbeit der beteiligten Autoren in der sorgfältigen Ursachensuche“, beschreibt Dr. Stefanie Keymel die Grundlagen ihrer Arbeit. Tatsächlich habe die Patientin selbst den zur Diagnose führenden Hinweis geliefert: Sie hat die Ausgusspräparate durch Fotografie dokumentiert. „Klinisch sehr interessant war die Zunahme des Abhustens der Ausgusspräparate bei Auftreten eines kardialen Ereignisses wie das Auftreten von Vorhofflimmern. Ein Zusammenhang zwischen der Bronchitis plastica und einer Herzerkrankung besteht bei Kindern mit angeborenen Herzfehlern und Fontanoperation. Ein Zusammenhang bei Erwachsenen wird selten beschrieben und hat uns motiviert, über den Fall zu berichten“, so die Preisträgerin. Dr. Stefanie Keymel ist Oberärztin am Universitätsklinikum Düsseldorf in der Klinik für Kardiologie, Pneumologie und Angiologie.

Der Fallbericht erschien in der OktoberAusgabe der Pneumologie (Pneumologie 2017; 71: 681-683).

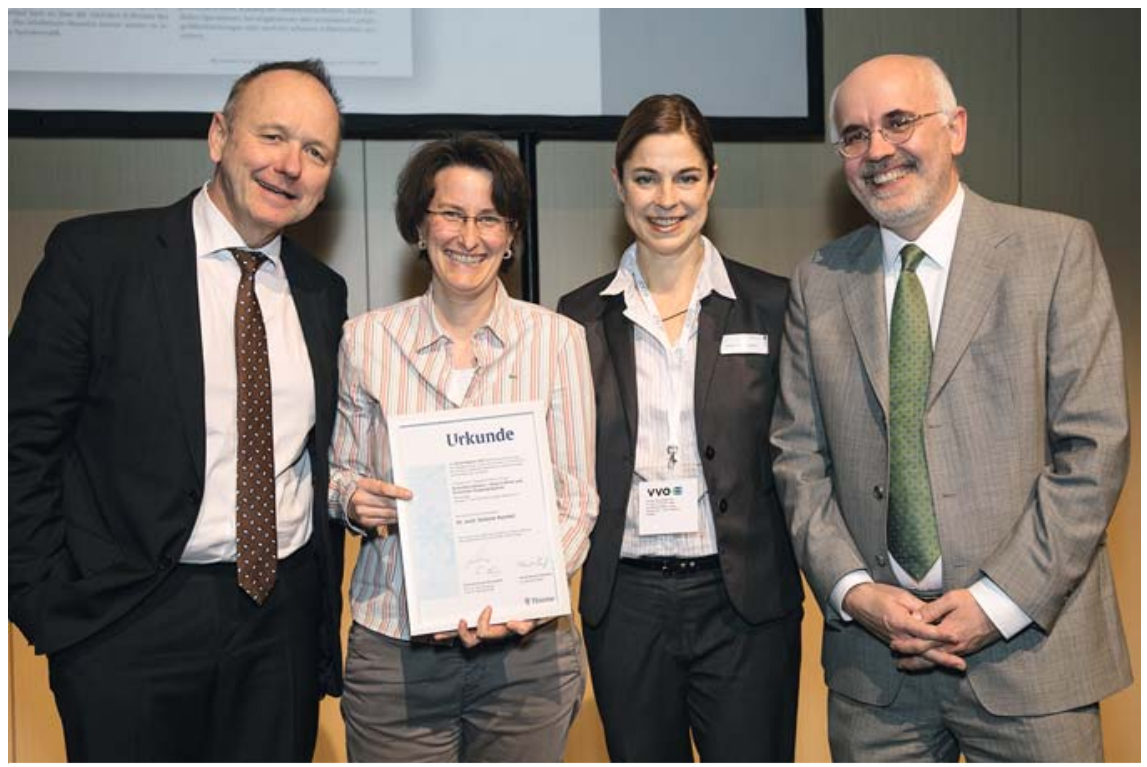

- Der Kasuistikpreis 2017 wurde im Rahmen des diesjährigen Kongresses der Deutschen Gesellschaft für Pneumologie und Beatmungsmedizin in Dresden verliehen.

Die Preisträgerin Dr. Stefanie Keymel (2.v.I) nahm die Glückwünsche vom DGP-Präsidenten Prof. Klaus F. Rabe, Sibylle Rettenmaier (Thieme Verlag) und dem Herausgeber der Pneumologie Prof. Santiago Ewig entgegen. Bildquelle: DGP/Mike Auerbach

Haben Sie auch einen interessanten Fall, über den Sie gerne berichten möchten? Dann schreiben Sie uns: Bewerbungen um den Kasuistikpreis 2018 können noch bis Ende des Jahres eingereicht werden bei Prof. Tom Schaberg unter Schaberg@diako-online.de. Der Kasuistikpreis ist mit einem Preisgeld von 500 Euro und einem Jahresabonnement der Zeitschrift verbunden.

Ihre Redaktion 